Kimmelmann, Nicole; Lang, Johannes

\title{
Lehramtsstudierende mit Migrationshintergrund und ihre Schwierigkeiten an
} der Universität

Seifried, Jürgen [Hrsg.]; Faßhauer, Uwe [Hrsg.]; Seeber, Susan [Hrsg.]: Jahrbuch der berufs- und wirtschaftspädagogischen Forschung 2014. Opladen [u.a.] : Verlag Barbara Budrich 2014, S. 135-146. (Schriftenreihe der Sektion Berufs- und Wirtschaftspädagogik der Deutschen Gesellschaft für Erziehungswissenschaft (DGfE); 217)

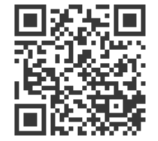

Quellenangabe/ Reference:

Kimmelmann, Nicole; Lang, Johannes: Lehramtsstudierende mit Migrationshintergrund und ihre Schwierigkeiten an der Universität - In: Seifried, Jürgen [Hrsg.]; Faßhauer, Uwe [Hrsg.]; Seeber, Susan [Hrsg.]: Jahrbuch der berufs- und wirtschaftspädagogischen Forschung 2014. Opladen [u.a.] : Verlag Barbara Budrich 2014, S. 135-146 - URN: urn:nbn:de:0111-pedocs-97315 - DOI: 10.25656/01:9731

https://nbn-resolving.org/urn:nbn:de:0111-pedocs-97315

https://doi.org/10.25656/01:9731

in Kooperation mit / in cooperation with:

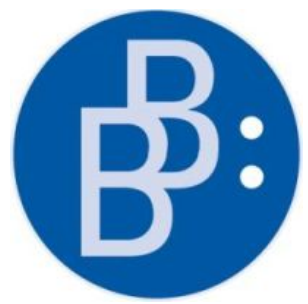

https://www.budrich.de

\section{Nutzungsbedingungen}

Dieses Dokument steht unter folgender Creative Commons-Lizenz: http://creativecommons.org/licenses/by-nc-nd/3.0/de/deed - Sie dürfen das Werk bzw. den Inhalt unter folgenden Bedingungen vervielfältigen, verbreiten und öffentlich zugänglich machen: Sie müssen den Namen des Autors/Rechteinhabers in der von ihm festgelegten Weise nennen. Dieses Werk bzw. dieser Inhalt darf nicht für kommerzielle Zwecke verwendet werden und es darf nicht bearbeitet, abgewandelt oder in anderer Weise verändert werden.

Mit der Verwendung dieses Dokuments erkennen Sie die Nutzungsbedingungen an.

\section{Terms of use}

This document is published under following Creative Commons-License: http://creativecommons.org/licenses/by-nc-nd/3.0/de/deed.en - You may copy, distribute and transmit, adapt or exhibit the work in the public as long as you attribute the work in the manner specified by the author or licensor. You are not allowed to make commercial use of the work or its contents. You are not allowed to alter, transform, or change this work in any other way.

By using this particular document, you accept the above-stated conditions of use.

\section{Kontakt / Contact:}

peDOCS

DIPF | Leibniz-Institut für Bildungsforschung und Bildungsinformation Informationszentrum (IZ) Bildung

E-Mail: pedocs@dipf.de

Internet: www.pedocs.de

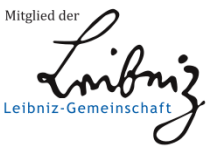




\section{Jahrbuch der berufs- und wirtschaftspädagogischen Forschung 2014}

Jürgen Seifried, Uwe Faßhauer Susan Seeber (Hrsg.)

DGE Deutsche Gesellschaft 
Schriftenreihe der Sektion

Berufs- und Wirtschaftspädagogik der Deutschen Gesellschaft für Erziehungswissenschaft (DGfE) 
Jürgen Seifried

Uwe Faßhauer

Susan Seeber (Hrsg.)

\section{Jahrbuch der berufs- und wirtschaftspädagogischen Forschung 2014}

Verlag Barbara Budrich

Opladen • Berlin • Toronto 2014 
Bibliografische Information der Deutschen Nationalbibliothek

Die Deutsche Nationalbibliothek verzeichnet diese Publikation in der Deutschen Nationalbibliografie; detaillierte bibliografische Daten sind im Internet über http://dnb.d-nb.de abrufbar.

(C) Dieses Werk ist im Verlag Barbara Budrich erschienen und steht unter folgender Creative Commons Lizenz: http://creativecommons.org/licenses/by-nc-nd/3.0/de/ Verbreitung, Speicherung und Vervielfältigung erlaubt, kommerzielle Nutzung und Veränderung nur mit Genehmigung des Verlags Barbara Budrich.

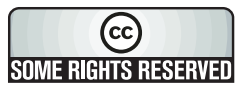

Dieses Buch steht im OpenAccess Bereich der Verlagsseite zum kostenlosen Download bereit (http://dx.doi.org/10.3224/84740164)

Eine kostenpflichtige Druckversion (Printing on Demand) kann über den Verlag bezogen werden. Die Seitenzahlen in der Druck- und Onlineversion sind identisch.

$$
\begin{array}{ll}
\text { ISBN } & 978-3-8474-0164-3 \text { (Paperback) } \\
\text { eISBN } & \mathbf{9 7 8 - 3 - 8 4 7 4 - 0 4 4 1 - 5 ~ ( e B o o k ) ~} \\
\text { DOI } & 10.3224 / 84740164
\end{array}
$$

Umschlaggestaltung: bettina lehfeldt graphic design, Kleinmachnow

Typografisches Lektorat: Judith Henning, Hamburg

Verlag Barbara Budrich, http://www.budrich-verlag.de 


\section{Inhaltsverzeichnis}

Vorwort 9

\section{Teil I: Perspektiven der historischen Berufsbildungsforschung}

Frank-Lothar Kroll

Möglichkeiten und Notwendigkeiten historiographischen Arbeitens in der Berufs- und Wirtschaftspädagogik........................................................ 1

Volker Bank, Annekathrin Lehmann

Theodor Franke. Sächsischer Pionier wirtschaftspädagogischen

Denkens in Deutschland

Marcel Schweder

Lehrerarbeit im Strafvollzug - Ein Diskurs aus historischer Sicht

\section{Teil II: Kompetenzmodellierung, -messung und -förderung}

Eveline Wittmann, Ulrike Weyland, Annette Nauerth, Ottmar Döring, Simone Rechenbach, Julia Simon, Iberé Worofka Kompetenzerfassung in der Pflege älterer Menschen - Theoretische und domänenspezifische Anforderungen der Aufgabenmodellierung 53

Simon Heinen, Martin Frenz, Christopher M. Schlick

Bildung für nachhaltige Entwicklung in der Gebäudeenergieberatung - Entwicklung eines Kompetenzmodells für die Förderung reflexiver Handlungsfähigkeit

Diana Stuckatz, Cornelia Wagner

Qualifizierungsangebote in der Pflegehilfe für Personen mit geringen Grundbildungskenntnissen - Empirische Studien zur Entwicklung von Lehr-Lern-Umgebungen und Arbeitsmaterialien. 81 


\section{Teil III: Gestaltung und Analyse von Lehr-Lern-Prozessen}

Eva Höpfer, Andrea Reichmuth, Doreen Holtsch, Franz Eberle

Wer sieht was? - Zum Umgang mit unterschiedlichen Sichtweisen auf

Unterricht am Beispiel des kaufmännischen Berufsschulunterrichts 95

Mandy Hommel

Sozial geteilte Reflexion - eine explorative Studie im

Mathematikunterricht. 109

Gerhard Minnameier, Rico Hermkes

„Kognitive Aktivierung“ und „konstruktive Unterstützung“ als Lehr-

Lern-Prozess-Größen - Eine Konzeption im rechnungswesen-

didaktischen Kontext

\section{Teil IV: Lehrerbildung und pädagogische Professionalität}

Nicole Kimmelmann, Johannes Lang

Lehramtsstudierende mit Migrationshintergrund und ihre

Schwierigkeiten an der Universität

Robert W. Jahn

Stützlehrer als neuer pädagogischer Profi in der Beruflichen Bildung?! .... 147

Sabrina Berg

Pädagogische Praxis und Reproduktion sozialer Ungleichheit - zur

Berücksichtigung sozialer Herkunft im Wirtschaftsunterricht..... 161

\section{Teil V: Perspektiven der Berufsbildungsforschung}

\section{Miriam Voigt}

Neo-institutionalistische und mikropolitische Prozesse in

Schulentwicklungsprojekten

Lara Forsblom, Lucio Negrini, Jean-Luc Gurtner \& Stephan Schumann

Lehrvertragsauflösungen und die Rolle der betrieblichen Auswahl von

Auszubildenden 
Marius R. Busemeyer

Organisierte Interessen, Parteipolitik und institutioneller Wandel im deutschen Berufsbildungssystem

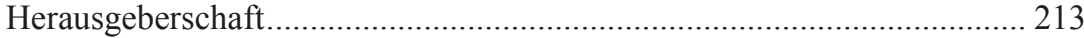

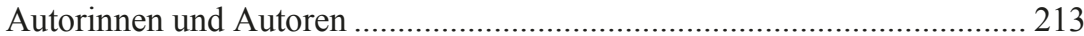




\title{
Lehramtsstudierende mit Migrationshintergrund und ihre Schwierigkeiten an der Universität
}

\author{
Nicole Kimmelmann, Johannes Lang
}

\section{Hintergrund}

Eine Reihe von Gründen spricht für das Streben nach einem hohen Anteil von Lehrkräften mit Migrationshintergrund. Hierzu zählen beispielsweise ihre Vorbildfunktion für Schüler mit Migrationshintergrund oder ihre Mehrsprachigkeit, welche Elternarbeit in vielen Fällen erleichtert (RangoschSchneck 2009, S. 3). Der reale Anteil dieser Lehrergruppe liegt an deutschen Schulen bei ca. 5 Prozent (Statistisches Bundesamt 2010, S. 259). Dem gegenüber steht ein deutlich höherer Anteil unter den Schülerinnen und Schülern. Beispielsweise liegt der Anteil der Schülerinnen und Schüler die einen Migrationshintergrund besitzen bei der für die Berufliche Bildung relevanten Altersgruppe der Fünfzehnjährigen bei nahezu 26 Prozent (Stanat/Rauch/Segeritz 2010, S. 224).

Um einen Beitrag zur Erhöhung des Anteils von Lehrkräften mit Migrationshintergrund zu leisten, muss untersucht werden, welche Hemmnisse auf dem Weg zum Lehrerdasein für diese Zielgruppe bestehen. Die höheren Studienabbruchquoten von Studierenden mit Migrationshintergrund (Bundesamt für Migration und Flüchtlinge 2011, S. 29) rücken dabei auch den Kontext der universitären Ausbildung selbst in den Fokus der Betrachtungen. Daraus ergibt sich für uns das Ziel, empirisch fundierte Aussagen über die Schwierigkeiten Studierender mit Migrationshintergrund im Vergleich zu Studierenden ohne Migrationshintergrund im Studium der Berufs- und Wirtschaftspädagogik treffen zu können. Der nachfolgend dargestellte Forschungsstand dient als Grundlage für die weitere Forschung. Er soll einen Überblick über die Bandbreite von Aspekten bieten, die möglicherweise den Studienerfolg von Studierenden mit Migrationshintergrund gefährden. Dabei handelt es sich um Problemfelder, die ausschließlich Studierende mit Migrationshintergrund oder alle Studierenden gleichermaßen betreffen können. Eingeschlossen sind auch Qualitätsmerkmale von Studiengängen (z. B. Erreichbarkeit von Lehrenden), deren subjektiv eingeschätzte Ausprägung Studierende mit Migrationshintergrund als problematisch wahrnehmen. 


\section{Methodisches Vorgehen}

Die Untersuchung des Forschungsproblems erfolgt mittels einer quantitativen Fragebogenerhebung bei Studierenden mit und ohne Migrationshintergrund, um Vergleiche zwischen den Gruppen ziehen zu können. Zur Ableitung der relevanten Items wurde der Forschungsstand systematisch erarbeitet. Die zu Beginn der Recherche eingesetzten Suchbegriffe fokussierten ausschließlich Lehramtsstudierende mit Migrationshintergrund. Der relativ geringe Umfang gefundener Literatur wurde zum Anlass genommen, weitere Suchbegriffe zu verwenden. So wurde auch die Literatur zu Studierenden mit Migrationshintergrund anderer Studiengänge einbezogen. Neben veröffentlichten Büchern, Beiträgen aus Sammelbänden und Zeitschriften fließt insbesondere Graue Literatur ein. Diese, vor allem in Form von ausgewerteten Studierendenumfragen einzelner Hochschulen, enthält ausführliche Informationen zum Forschungsgegenstand. Durchsucht wurden die Datenbanken Fachinformationssystem Bildung, World Cat, Google Scholar sowie OPACplus der FriedrichAlexander-Universität Erlangen-Nürnberg (inklusive des Bestands des Bibliothekverbundes Bayern). Die verwendeten Suchbegriffe und Suchprofile können der nachfolgenden Tabelle entnommen werden.

\section{Tab. 1: Suchbegriffe und Suchprofile}

\begin{tabular}{ll}
\hline Datenbanken: & FIS Bildung, OPACplus FAU Erlangen-Nürnberg, WorldCat \\
\hline Suchbegriffe: & Lehramtsstudium Migrationshintergrund; Lehramtsstudenten \\
& $\begin{array}{l}\text { Migrationshintergrund; Lehramtsstudierende Migrationshinter- } \\
\text { grund; Lehramtsstudierende ,kulturelle Vielfalt“; Studium }\end{array}$ \\
& Migrationshintergrund; Studierende Migrationshintergrund; \\
& Studenten Migrationshintergrund; ,, Phase der Lehrerbildung“ \\
& Migrationshintergrund; Lehrerbildung Migrationshintergrund; \\
& Universität Migrationshintergrund; Hochschule Migrationshin- \\
& tergrund; Probleme Studierende Migrationshintergrund; \\
& Schwierigkeiten Studierende Migrationshintergrund; Probleme \\
& Studenten Migrationshintergrund \\
Verknüpfung: & AND \\
Suchprofil: & Freitext \\
Zeitraum: & Keine Einschränkung \\
Sprache: & Deutsch \\
Zeitpunkt: & 07.05.2013 \\
Treffer: & FIS Bildung: 545 \\
& OPACplus FAU Erlangen-Nürnberg: 446 \\
& WorldCat: 421 \\
\hline
\end{tabular}


Tab. 1: Suchbegriffe und Suchprofile (Fortsetzung)

\begin{tabular}{ll}
\hline Datenbank: & Google Scholar \\
\hline Suchbegriffe: & $\begin{array}{l}\text { "Lehramtsstudenten mit Migrationshintergrund“; „Lehramts- } \\
\text { studierende mit Migrationshintergrund“; ,Studierende mit } \\
\end{array}$ \\
& $\begin{array}{l}\text { Migrationshintergrund“; ,Studenten mit Migrationshinter- } \\
\text { grund“ }\end{array}$ \\
& Genaue Wortfolge \\
Verknüpfung: & Freitext \\
Suchprofil: & Keine Einschränkung \\
Zeitraum: & Deutsch \\
Sprache: & 08.05 .2013 \\
Zeitpunkt: & 244 \\
Treffer: &
\end{tabular}

Google Scholar lieferte bei Eingabe der für die anderen Datenbanken verwendeten Suchbegriffe (unter Verwendung des gleichen Suchprofils) eine fünfstellige Trefferzahl. Daher wurden hierfür gesonderte Suchbegriffe (und ein abweichendes Suchprofil) verwendet. Insgesamt wurden nach Filterung der Redundanzen und themenfremden Veröffentlichungen 19 Publikationen als relevant eingestuft, da sie Fragen behandeln, die für die Bearbeitung des Forschungsproblems von Interesse sind und neue Ansatzpunkte liefern (vgl. Hofmann 2013, S. 101). Diese Publikationen begründen den für die Datenerhebung zugrundegelegten Forschungsstand. Um diesen übersichtlich darstellen zu können, wurden induktiv Kategorien gebildet (vgl. Bortz/Döring 2006, S. 151). Da möglichst alle relevanten Faktoren erfasst werden sollten, um sie in ihrer Bedeutung für die Wirtschafts- und Berufspädagogik abfragen zu können, fand keine Limitierung der Items mit Blick auf die Häufigkeit des Auftretens oder ihrer Bedeutung in der Literatur statt. Wo vorhanden, werden vergleichende Gegenüberstellungen mit Studierenden ohne Migrationshintergrund vorgenommen. Diese stammen jedoch z. T. aus Studien, die als nicht repräsentativ einzustufen sind.

\section{Ergebnisse}

\subsection{Sprachlich-kommunikative Schwierigkeiten}

Studierende mit Migrationshintergrund schätzen ihre schriftlichen Deutschkenntnisse schlechter ein als Studierende ohne Migrationshintergrund (Karakasoglu 2011, S. 129). Einem Anteil der Studierenden mit Migrationshintergrund werden sogar Sprachdefizite attestiert. So haben Studierende Probleme im Umgang mit Fachsprache (Hopp/Drüke 2008, S. 36, Naumann 2011, 
S. 21, Meinhardt/Zittlau 2009, S. 98). Passend dazu äußern Studierende den Wunsch nach Fachsprachtraining (Karakasoglu 2010, S. 30). Auch von Problemen, wissenschaftlich zu schreiben, wird berichtet (Hopp/Drüke 2008, S. 36f, Meinhardt/Zittlau 2009, S. 99). In Verbindung damit können die festgestellten orthographischen und grammatischen Defizite gesehen werden (Hopp/Drücke 2008, S. 37, Meinhardt/Zittlau 2009, S. 98f.), sowie der Wunsch Studierender nach Ausweitung studienbegleitender Förderangebote zur Vermittlung orthographischer und grammatischer Kompetenzen (Caglayan-Babursah/Chrissou 2000, S. 40). Möglicherweise lassen sich defizitäre Kenntnisse der deutschen Sprache teilweise dadurch erklären, dass ein Anteil der Studierenden mit Migrationshintergrund mit der eigenen Familie überwiegend nicht Deutsch spricht und auch im Freundeskreis eine andere Sprache verwendet (Müller/Kellmer 2011, S. 18).

\subsection{Diskriminierung, Vorurteile und fehlende Wertschätzung}

Einige Studierende mit Migrationshintergrund fordern eine Atmosphäre der Wertschätzung kultureller Vielfalt an den Hochschulen, insbesondere die Wahrnehmung von Mehrsprachigkeit als Bereicherung (Hopp/Drüke 2008, S. 49). Berichtet wird in diesem Zusammenhang davon, dass Studierende mit Migrationshintergrund als Repräsentanten einer ,,anderen“ Kultur angesprochen werden, wodurch eine Bestärkung der Andersheit erfolgt (Discher/Plößer 2010, S. 7f.). Studierende mit Migrationshintergrund erfahren Diskriminierungen durch andere Studierende und sind Vorurteilen ausgesetzt (Meinhardt/Zittlau 2009, S. 77f.). So wird von Beleidigungen und ausgrenzendem Verhalten gegenüber Studierenden mit anderer nationaler Herkunft berichtet (Müller/Kellmer 2011, S. 15), außerdem von Rassismuserfahrungen, ausgelöst durch Kommentare von Kommilitonen (Discher/Plößer 2010, S. 12f.) und von dem Gefühl aufgrund des Tragens eines Kopftuches von Kommilitonen abgelehnt zu werden (Hopp/Drüke 2008, S. 51). Einige Studierende haben das Gefühl aufgrund ihrer Herkunft von Lehrpersonal in Lehrveranstaltungen und bei der Notengebung ungerecht behandelt zu werden sowie von Vorurteilen von Lehrpersonal betroffen zu sein (Müller/Kellmer 2011, S. 15). Es wird von der diskriminierenden Formulierung von Aufgabenstellungen (Discher/Plößer 2010, S. 19, Karakasoglu et al. 2013, S. 70f.) und von herkunftsbezogener Zuschreibung geringer Leistungsfähigkeit berichtet (ebd., S. 71, Meinhardt/Zittlau 2009, S. 77f.). Weiterhin existiert bei Studierenden das Gefühl, aufgrund des Tragens eines Kopftuches von Lehrpersonal diskriminiert zu werden (Hopp/Drüke 2008, S. 52). Bezüglich der Verwaltungsangestellten existieren Aussagen einer Gruppe Studierender mit Migrationshintergrund, wonach diese sich von den Angestellten überwiegend unfreundlich behandelt fühlen (Meinhardt/Zittlau 2009, S. 109f.). Außerdem 
geben Studierende an, als Sonderlinge behandelt zu werden und vermuten, dass sie bei der Vergabe von Stellen als studentische Hilfskräfte aufgrund ihres Migrationshintergrundes benachteiligt werden (ebd., S. 110). Hinzu kommen insbesondere zu Beginn des Studiums Schwierigkeiten aufgrund religiöser Gewohnheiten (ebd., S. 86).

Studierende mit Migrationshintergrund sind auch außerhalb der Universität (z. B. in Schulpraxiseinsätzen) Stereotypisierungen ausgesetzt beispielsweise aufgrund ihres Kopftuches - was sich auf ihren Studienerfolg auswirken kann (Meinhardt/Zittlau 2009, S. 112, Hopp/Drüke 2008, S. 53, Karakasoglu et al. 2013, S. 71).

\subsection{Soziale Integration und Zusammenarbeit mit Lehrpersonal und autochthonen Kommilitonen}

Studierende mit Migrationshintergrund haben häufiger als andere Studierende wenig Kontakt zu Kommilitonen (Kurré et al. 2011, S. 5, Meinhardt/Zittlau 2009, S. 107). Unter ihnen besteht die Tendenz, sich mehr mit Studierenden mit Migrationshintergrund zu umgeben als mit autochthonen Studierenden (Meinhardt/Zittlau 2009, S. 107, Hopp/Drüke 2008, S. 45). Als Resultat dessen kann das mangelnde Zugehörigkeitsgefühl einiger Studierender mit Migrationshintergrund gesehen werden (Naumann 2011, S. 23). Sprachdefizite hemmen Studierende in der Kontaktaufnahme zu Kommilitonen und führen zu Ausschließungsprozessen und verwehrter sozialer Anerkennung (Discher/Plößer 2010, S. 9f.). Geringe soziale Integration bedeutet gleichzeitig fehlende soziale Netzwerke innerhalb der Hochschule, welche zur Überwindung von Studienproblemen genutzt werden könnten (Meinhardt/Klausing 2009, S. 73). Konsistent dazu beklagen einige Studierende mit Migrationshintergrund mangelnde Unterstützung und fehlende Hilfestellung durch andere Studierende (Kurré et al. 2011, S. 5, Meinhardt/Zittlau 2009, S. 77). Nach den Aussagen einiger Studierender wird das soziale Klima aufgrund der herrschenden Konkurrenzsituation negativ beeinflusst (ebd., S. 108). Auch in Arbeitsgruppen verläuft die Zusammenarbeit der Studierenden nicht immer problemlos (ebd., S. 99). So entstehen durch unterschiedliche Lebensbedingungen Kooperationsprobleme (Naumann 2011, S. 23) und Studierende mit Migrationshintergrund erfahren soziale Ausschlussprozesse im Kontext von Gruppenarbeiten (Discher/Plößer 2010, S. 6).

Von einigen Studierenden mit Migrationshintergrund wird die schlechte Erreichbarkeit der Hochschullehrenden moniert (Meinhardt/Zittlau 2009, S. 91) und die geringe Anzahl der angebotenen Sprechstunden kritisiert (ebd., S. 121). Ebenfalls nicht durchweg positiv bewertet wird die Bereitschaft der Lehrenden, auf erbrachte Leistungen Feedback zu geben und auf Fragen und Probleme der Studierenden einzugehen (ebd., S. 92). Einige Studierende mit 
Migrationshintergrund sprechen sogar von Unfreundlichkeit und Desinteresse seitens Lehrender (ebd., S. 92).

\subsection{Finanzierung}

Im Vergleich mit autochthonen Studierenden stehen Studierenden mit Migrationshintergrund durchschnittlich weniger finanzielle Mittel zur Verfügung (Hopp/Drüke 2008, S. 44, Isserstedt et al. 2010, S. 510). Sie haben häufiger einen niedrigeren sozio-ökonomischen Hintergrund als Studierende ohne Migrationshintergrund (Tepecik 2010, S. 27, Rokitte 2012, S. 23), werden seltener (Müller/Kellmer 2011, S. 31) und in geringerem Umfang (Isserstedt et al. 2010, S. 510) von ihren Eltern finanziell unterstützt und müssen häufiger andere Personen mitfinanzieren (Decker et al. 2010, S. 7, Müller/Kellmer 2011, S. 32). Studierende mit Migrationshintergrund beziehen häufiger BAföG (Berthold et al. 2012, S. 18, Kurré et al. 2011, S. 4) und BAföG macht bei ihnen einen höheren Anteil des Einnahmebudgets aus (Karakasoglu 2011, S. 129, Isserstedt et al. 2010, S. 510) als bei Studierenden ohne Migrationshintergrund. Außerdem ist der Anteil der Einnahmen aus eigenem Verdienst bei Studierenden mit Migrationshintergrund höher als bei Studierenden ohne Migrationshintergrund (Isserstedt et al. 2010, S. 510), sie sind neben dem Studium häufiger (Decker et al. 2010, S. 7, Kurré et al. 2011, S. 4) und in höherem Umfang (Decker et al. 2010, S. 7, Berthold et al. 2012, S. 18) erwerbstätig. Über den Anteil, der ein Stipendium erhält, lässt sich nur wenig aussagen. Naumann (2011, S. 20) stellt weit verbreitete Unwissenheit über die Förderungsmöglichkeit durch Stipendien fest. Rokitte (2012, S. 26) attestiert, dass der Anteil geförderter Studierender mit Migrationshintergrund, vor allem derer mit niedriger sozialer Herkunft, sehr gering ausfällt.

Passend dazu geben mehr Studierende mit Migrationshintergrund als Studierende ohne Migrationshintergrund an, ihr Studium aufgrund finanzieller Probleme unterbrochen $\mathrm{zu}$ haben (Ihsen/Hantschel/Hackl-Herrwetth/Wienefort/Baldin 2010, S. 16, Karakasoglu 2013, S. 149, Isserstedt et al. 2010, S. 510, Rokitte 2012, S. 22). Der hohe für das Arbeiten betriebene zeitliche Aufwand verursacht Kooperationsprobleme mit Kommilitonen (Naumann 2011, S. 23). Außerdem führen fehlende finanzielle Mittel zu verringerter Partizipation an erweiterten Ausbildungsmöglichkeiten wie der Durchführung eines Auslandssemesters (Rokitte 2012, S. 23). Begünstigt werden zusätzlich Schwierigkeiten beim Knüpfen von Kontakten zu anderen Studierenden, da abendliches Ausgehen nicht finanziert werden kann (Meinhardt/Zittlau 2009, S. 108). 


\subsection{Zeitinvestition und Wohnen}

Zeitliche Probleme schlagen sich im Studienverlauf nieder. Einige Studierende mit Migrationshintergrund liegen hinter den Vorgaben der Studienordnung zurück, teilweise um mehrere Semester (Meinhardt/Zittlau 2009, S. 101f.). Im Zusammenhang damit steht die geäußerte Kritik an hohen Präsenzzeiten und hohem Arbeitsaufwand (ebd., S. 124).

Dabei wohnen Studierende mit Migrationshintergrund häufiger bei ihren Eltern als Studierende ohne Migrationshintergrund (Karakasoglu 2010, S. 514). Die für das Studium zur Verfügung stehende Zeit wird dann bei einigen Studierenden mit Migrationshintergrund durch das Pendeln zwischen Wohnort und Studienort reduziert (Decker et al. 2010, S. 7, Meinhardt/Zittlau 2009, S. 114). Hinsichtlich der Zufriedenheit mit ihrer Wohnsituation lassen die Aussagen einiger Studierender mit Migrationshintergrund den Schluss zu, dass auch Bewohner von Studentenwohnheimen teilweise schwierigen Wohnbedingungen ausgesetzt sind (Meinhardt/Zittlau 2009, S. 79).

\subsection{Curriculares Angebot und Prüfungsleistungen}

Studierende mit Migrationshintergrund berichten von Lehrveranstaltungen die nicht besucht bzw. belegt werden können, da sie überfüllt sind oder sich zeitlich überschneiden (Meinhardt/Zittlau 2009, S. 118). Beratungsinstanzen weisen darauf hin, dass ungenügend abgestimmte Lehrveranstaltungen Hürden für den Studienerfolg darstellen können (Caglayan-Babursah/Chrissou 2000, S. 34). Seitens der Studierenden werden strikte Vorgaben in der Studienstruktur ohne Freiraum für selbstständige Studiengestaltung sowie unzureichend qualifizierte Tutoren bemängelt (Meinhardt/Zittlau 2009, S. 116f.). Der praktische Bezug und die Qualität der Lehrveranstaltungen wird von einigen Studierenden mit Migrationshintergrund schlecht bewertet, ebenso das Bemühen der Hochschullehrer um eine verständliche Darstellung der Inhalte (ebd., S. 89f.). Es kommt sogar das Gefühl zum Ausdruck, an der Universität nicht wirklich ausgebildet zu werden (ebd., S. 106). Als weitere Probleme im Rahmen von Vorlesungen werden mangelnde Fachkenntnisse und hohes Tempo bei der Vermittlung von Inhalten genannt (Hopp/Drüke 2008, S. 36).

Nicht unerheblich ist die Anzahl der Studierenden mit Migrationshintergrund, die sich den Leistungsanforderungen im Studium nicht gewachsen fühlen (Meinhardt/Zittlau 2009, S. 97). Passend dazu wird die Angst Studierender beschrieben, Prüfungen und Klausuren nicht zu bestehen (ebd., S. 98). Vielen Studierenden mit Migrationshintergrund fehlen im privaten Umfeld Personen, die bei fachlichen Problemen und bei der Erstellung wissenschaftlicher Arbeiten Unterstützung leisten können (Hopp/Drüke 2008, S. 29, Naumann 2011, S. 21). Probleme beim Schreiben wissenschaftlicher Arbei- 
ten bereitet die schriftliche Darstellung von Arbeitsergebnissen (Meinhardt/ Zittlau 2009, S. 99). Gleichzeitig besteht Unsicherheit bzw. Unwissenheit über Prinzipien des wissenschaftlichen Schreibens (Caglayan-Babursah/ Chrissou 2000, S. 40, Hopp/Drüke 2009, S. 36), beispielsweise über systematisches Vorgehen zur Recherche von Informationen (Meinhardt/Zittlau 2009, S. 99).

Die Vorbereitung, Begleitung und Reflexion von Praktika und Praxissemestern wird von einem Großteil der Studierenden mit Migrationshintergrund als schlecht empfunden (ebd., S. 90). Von ihnen werden der zu geringe Umfang von Schulpraktika sowie der dabei geringe Anteil praktischer Tätigkeiten kritisiert (Caglayan-Babursah/Chrissou 2000, S. 36).

\subsection{Informationsdefizite}

Ein großes Informationsdefizit ist unter Studierenden mit Migrationshintergrund bezüglich der Studienfinanzierung über Stipendien vorhanden. So ist der Bekanntheitsgrad der Studienstipendien der Begabtenförderwerke extrem niedrig (Naumann 2011, S. 20, Rokitte 2012, S. 26). Das Weiterbildungsangebot ist auch nach mehreren Semestern vielen Studierenden unbekannt (Meinhardt/Zittlau 2009, S. 97). Eklatant ist zudem die Unbekanntheit einiger Beratungs- und Unterstützungsangebote (Hopp/Drüke 2008, S. 30, Meinhardt/Zittlau 2009, S. 93f.). Vor Studienbeginn sind die Studierenden häufig schlecht über die Studienbedingungen informiert. Dies betrifft die Leistungsanforderungen im Studium, Möglichkeiten zur Studienfinanzierung, Prüfungsmodalitäten, Lernbedingungen sowie Wohnmöglichkeiten (Meinhardt/Zittlau 2009, S. 66). Nach mehreren Semestern bestehen immer noch Informationsdefizite bezüglich der Prüfungsvorschriften (ebd., S. 88f.) und es existieren Unsicherheiten bei der Auslegung der Studienordnung (CaglayanBabursah/Chrissou 2000, S. 36). Weitere Informationsdefizite betreffen die Formalien zur Anmeldung für den Vorbereitungsdienst, dessen Strukturierung und Anforderungen (ebd., S. 34ff.). Dies ist den Studierenden durchaus bewusst. Sie fühlen sich nicht ausreichend informiert und kritisieren die Informationsvermittlung diesbezüglich (ebd., S. 35f.).

\subsection{Außercurriculares Hochschulangebot, selbstständige Studienorganisation und Erfolgsdruck}

Entsprechend der bereits erwähnten Unbekanntheit mehrerer Beratungs- und Unterstützungsangebote ist auch deren Inanspruchnahme äußerst gering (Caglayan-Babursah/Chrissou 2000, S. 36, Meinhardt/Zittlau 2009, S. 93f.). Gleiches gilt für Angebote zur Weiterbildung bzw. der Qualifizierung über das Fachstudium hinaus (ebd., S. 94f.). Die Qualität von Beratungsangeboten 
bewerten die Studierenden mit Migrationshintergrund als teilweise schlecht. Es werde ihnen nicht angemessen weitergeholfen (Caglayan-Babursah/Chrissou 2000, S. 36) und die Berater seien teilweise unwissend oder überfordert (Meinhardt/Zittlau 2009, S. 120). In einigen Fällen wird die schlechte Erreichbarkeit von Beratungsstellen und Ämtern geschildert (ebd., S. 122). Außerdem wird das Gefühl geäußert, die Berater zu belästigen und von diesen abgefertigt zu werden, so dass kein Vertrauen aufgebaut werden kann (Caglayan-Babursah/Chrissou 2000, S. 36).

Studierende mit Migrationshintergrund wechseln häufiger den Studiengang und unterbrechen häufiger ihr Studium (Isserstedt et al. 2010, S. 509f.). Zudem sehen sie häufiger eigenen Unterstützungsbedarf bei Arbeitsorganisation, Zeitmanagement, Studienaufbau und -planung (Karakasoglu 2010, S. 30). Probleme bereitet Studierenden die selbstständige Planung und Organisation des Studiums (Hopp/Drüke 2008, S. 33, Meinhardt/Klausing 2009, S. 12, Meinhardt/Zittlau 2009, S. 100) sowie die Bewältigung bürokratischer Aufgaben (Naumann 2011, S. 23). Zudem wünschen Studierende Beratung zu den Themen Finanzierung, Vereinbarkeit von Familie und Studium sowie von Erwerbstätigkeit und Studium (Berthold et al. 2012, S. 19). Zu den Problemen der selbstständigen Studienorganisation kommt bei einigen Studierenden Erfolgsdruck durch ihre Eltern hinzu (Hopp/Drüke 2008:31). Dabei fehlt es vor allem Eltern ohne akademischen Abschluss an Verständnis für die Studiensituation ihrer Kinder (Meinhardt/Zittlau 2009, S. 136f.).

Eine Folge der weiter oben beschriebenen durchschnittlich geringeren zur Verfügung stehenden finanziellen Mittel ist die fehlende Partizipation erweiterter Studienangebote, beispielsweise die Durchführung von Auslandsaufenthalten (Rokitte 2012, S. 25f.). Für geplante Auslandsaufenthalte wünschen sich die Studierenden mehr Unterstützung (Meinhardt/Zittlau 2009, S. 126).

\section{Ausblick}

Um empirisch fundierte Aussagen über die Probleme von Studierenden mit Migrationshintergrund der Wirtschafts- und Berufspädagogik treffen zu können, müssen, wie gezeigt wurde, eine Vielzahl an Faktoren untersucht werden. Um die unterschiedliche Höhe der Studienabbruchquoten bei Studierenden mit und ohne Migrationshintergrund begründen zu können, dürfen nicht ausschließlich die Schwierigkeiten der Studierenden mit Migrationshintergrund betrachtet werden. Viele der ermittelten Faktoren können auch für Studierende ohne Migrationshintergrund Hürden darstellen. Deshalb gilt es, mittels Vergleich signifikante Unterschiede zwischen beiden Studierendengruppen zu ermitteln. Hierfür soll ein, auf Basis dieses Reviews entwickelter Fra- 
gebogen beide Zielgruppen erfassen. Nicht möglich ist ein Vergleich zwischen den beiden Studierendengruppen zu den Aspekten, die Diskriminierung und Vorurteile aufgrund einer nicht-deutschen Herkunft thematisieren (vgl. Abschnitt 3.2).

Prinzipiell ist zudem zu hinterfragen ob Unterschiede im Studienerfolg tatsächlich nur aufgrund der Zugehörigkeit zum Konstrukt „Migrationshintergrund" begründet und untersucht werden sollten. Betrachtet man deutsche Studierende so sind vor allem die Personen mit bildungsfernem Elternhaus in der Studierendenschaft unterrepräsentiert (vgl. Gwosć et al. 2011, S. 11). Bei einem Großteil der Personen mit Migrationshintergrund in Deutschland handelt es sich um Nachfahren von Gastarbeitern und somit mehrheitlich um Personen die aus eher bildungsfernen Elternhäusern stammen. Daher scheint es sinnvoll, auch Studierende aus bildungsfernen Elternhäusern verstärkt in den Fokus des Interesses zu rücken, um Studienerfolg von der Bildungslaufbahn der Eltern zu entkoppeln.

\section{Literatur}

Berthold, C./Leichsenring, H./Brandenburg, U./Güttner, A./Kreft, A./Morzick, B./ Noe, S./Reumschüssel, E./Schmalreck, U./Willert, M./Berghoff, S. (2012): CHE Diversity Report C2: Lehramt. CHE Consult GmbH. Online: http://www.checonsult.de/downloads/CHE_DiversityReport C2.pdf (19-07-2013).

Bortz, J./Döring, N. (2006): Forschungsmethoden und Evaluation (4. Auflage). Heidelberg: Springer Medizin.

Bundesamt für Migration und Flüchtlinge (2011): Integration von Studierenden mit Migrationshintergrund an deutschen Hochschulen: Bestandsaufnahme und Vernetzung.Online: http://www.bamf.de/SharedDocs/Anlagen/DE/Publikationen/Broschueren/broschuere-integration-studierende-hochschule.pdf?_blo$\mathrm{b}=$ publicationFile (15-08-2013).

Caglayan-Babursah, S./Chrissou, M. (2000): Qualifizierung von Studierenden mit Migrationshintergrund. Deutsch lernen, 25. Bd., H. 1, S. 32-43.

Decker, P./Pierdzioch, C./Stadtmann, G. (2010): Erfolgreich VWL studieren: Ausländische Männer haben`s schwer - nimm`s leicht? Europa-Universität Viadrina: Lehrstuhl für Volkswirtschaftslehre. Online: http://www.wiwi.europa-uni.de/de/ lehrstuhl/fine/makro/forschung/Klausurerfolg_Decker_06_10_2010.pdf (19-072013).

Discher, K./Plößer, M. (2010): Ergebnisbericht: „Erfahrungen von Studierenden mit Migrationshintergrund an der Fachhochschule Kiel“. Fachhochschule Kiel: Institut für Interdisziplinäre Genderforschung und Diversity. Online: http:// www.fh-kiel.de/fileadmin/data/technologietransfer/institut_frauenforschung/ pdfs/Ergebnisbericht_fertig1.doc. pdf (18-07-2013). 
Gwosć, C./Netz, N./Orr, D./Middendorff, E./Isserstedt, W. (2011): Soziale und wirtschaftliche Bedingungen des Studiums: Deutschland im europäischen Vergleich Eurostudent IV 2008-2011. Bielefeld: Bertelsmann.

Hofmann, J. (2013): Erfolgreich recherchieren - Erziehungswissenschaften. Berlin: De Gruyter.

Hopp, M./Drüke, S. (2008): Förderbedarfe und Förderungswünsche von Lehramtsstudentinnen mit Migrationshintergrund. Essen: Zentrum für Lehrerbildung.

Ihsen, S./Hantschel, V./Hackl-Herrwetth, A./Wienefort, V./Baldin, D. (2010): Ingenieurwissenschaften: Attraktive Studiengänge und Berufe auch für Menschen mit Migrationshintergrund?. Technische Universität München: Fachgebiet Gender Studies in Ingenieurwissenschaften. Online: http://4ing-online.de/fileadmin/ uploads/pdf/ThemenProjekte/20100520_Migrationsstudie.pdf(19-07-2013).

Isserstedt, W./Middendorff, E./Kandulla, M./Borchert, L./Leszczensky, M. (2010): Die wirtschaftliche und soziale Lage der Studierenden in der Bundesrepublik Deutschland 2009: 19. Sozialerhebung des Deutschen Studentenwerkes durchgeführt durch HIS Hochschul-Informations-System. Berlin: Bundesministerium für Bildung und Forschung. Online: http://www.sozialerhebung.de/download/ 19/Soz19_Haupt_Internet_A5.pdf (19-07-2013).

Karakasoglu, Y. (2010): Was macht den Unterschied? Studierende mit und ohne Migrationshintergrund: Aktueller Forschungsstand und Handlungserfordernisse. Vortrag im Rahmen der Arbeitskonferenz ,Studierende mit Migrationshintergrund" in Hannover, 08.02.2010. Online: http:/www.studieren-inniedersachsen.de/veranstaltungen/ 02-2010-arbeitskonferenz-migrationshintergrund/Karakasoglu.pdf (18-07-2013).

Karakasoglu, Y. (2011): Lehrer, Lehrerinnen und Lehramtsstudierende mit Migrationshintergrund: Hoffnungsträger der interkulturellen Öffnung von Schule. In: Neumann, U./Schneider, J. (Hrsg.): Schule mit Migrationshintergrund. Münster: Waxmann, S. 121-135.

Karakasoglu, Y. (2013): Integration durch Bildung - welche Wege sind zu beschreiben? In Brinkmann, H. U./Uslucan, H.-H. (Hrsg.): Dabeisein und Dazugehören: Integration in Deutschland. Wiesbaden: Springer Fachmedien, S. 127148.

Karakasoglu, Y./Wojciechowicz, A./Bandorski, S./Kul, A. (2013): Zur Bedeutung des Migrationshintergrundes im Lehramtsstudium: Quantitative und qualitative Grundlagenstudie und Reflexion von Praxismaßnahmen an der Universität Bremen. Universität Bremen: Arbeitsbereich Interkulturelle Bildung. Online: http://www.fb12.uni-bremen.de/fileadmin/Arbeitsgebiete/interkult/Publikationen/ Bedeutung_Migrationshintergrund_Lehramtsstudium_Stand_AK18.05..pdf (1807-2013).

Kurré, J./Scholl, J./Bullinger, M./Petersen-Ewert, C. (2011): Integration and healthrelated quality of life of undergraduate medical students with migration backgrounds - Results of a survey. GMS Psycho-Social-Medicine, 2011 8. Bd., S. 18 .

Lienert, G. A. (1969): Testaufbau und Testanalyse (3. Auflage). Weinheim: Beltz.

Meinhardt, R./Klausing, J. (2009): Hochschullotsen an der Universität Oldenburg: Evaluierung eines Pilotprojektes. Schriftenreihe des Interdisziplinären Zentrums für Bildung und Kommunikation in Migrationsprozessen (IBKM) an der Carl von Ossietzky Universität Oldenburg: 47. Oldenburg: BIS-Verlag. 
Meinhardt, R./Zittlau, B. (2009): BildungsinländerInnen an deutschen Hochschulen am Beispiel der Universität Oldenburg: Eine empirische Studie zu den erfolgshemmenden Faktoren im Studienverlauf und Empfehlungen zur Verbesserung der Studienleistungen durch HochschullotsInnen. Schriftenreihe des Interdisziplinären Zentrums für Bildung und Kommunikation in Migrationsprozessen (IBKM) an der Carl von Ossietzky Universität Oldenburg: 42. Oldenburg: BISVerlag.

Müller, U./Kellmer, A. (2011): Studierende mit Migrationshintergrund: Ergebnisse der großen UDE-Studierendenbefragung. Universität Duisburg-Essen: Zentrum für Hochschul- und Qualitätsentwicklung. Online: www.uni-due.de/imperia/md/ content/diversity/ude-studierendenbefragung_migrationshintergrund_20.12.2011 f.pdf (18-07-2013).

Naumann, I. (2011): Lehramtsstudierende mit Migrationshintergrund an der Universität Kassel: Eine Analyse qualitativer Interviews im Rahmen des Projektes „Mentoring für Lehramtsstudierende mit Migrationshintergrund“. Universität Kassel: Studiencenter Lehre. Online: http://www.mentoring-mig.uni-kassel.de/ wp-content/uploads/2011/11/forschungsbericht_mentoring_naumann_end.pdf (18-07-2013).

Rangosch-Schneck, E. (2009): Lehrpersonen mit Migrationshintergrund: RollenBilder-Potentiale. In: Landeshauptstadt Stuttgart (Hrsg.): Migranten machen Schule! Vielfalt im Klassenzimmer. Portfolio. Online: http://www.stuttgart.de/ item/show/393070/1 (14-06-2013).

Rokitte, R. (2012): Studierende mit Migrationshintergrund und Interkulturalität: Expertise im Rahmen des Projektes „Chancengleichheit in der Begabtenförderung" der Hans-Böckler Stiftung. Hans-Böckler-Stiftung Arbeitspapier, Bildung und Qualifizierung: 248. Online: http://www.boeckler.de/pdf/p_arbp_ 248.pdf (19-07-2013).

Schnell, R./Hill, P. B./Esser, E. (2008): Methoden der empirischen Sozialforschung (8. Auflage). München: Oldenbourg.

Stanat, P./Rauch, D./Segeritz, M. (2010): Schülerinnen und Schüler mit Migrationshintergrund. In: Klieme, E./Artelt, C./Hartig, J./Jude, N./Köller, O./Prenzel, M./Schneider, W./Stanat, P (Hrsg.): Pisa 2009: Bilanz nach einem Jahrzehnt. Münster: Waxmann, S. 200-230.

Statistisches Bundesamt (2010): Mikrozensus 2008: Personen mit Migrationshintergrund unter den Erwerbstätigen mit Hochschulabschluss und unter den Lehrkräften. In Autorengruppe Bildungsberichterstattung (Hrsg.): Bildung in Deutschland 2010 - Ein indikatorengestützter Bericht mit einer Analyse zu Perspektiven des Bildungswesen im demografischen Wandel. Bielefeld: W. Bertelsmann.

Tepecik, E. (2010): Bildungserfolge mit Migrationshintergrund: Biographien bildungserfolgreicher Migrantinnen türkischer Herkunft. Wiesbaden: VS Verlag für Sozialwissenschaften.

Zwengel, A. (2012): Studium interkulturell. Bildungsinländer und Bildungsausländer im Vergleich. Die Hochschule, 21. Bd., H. 1, S. 55-72. 